\title{
Cólico renal: diagnóstico y tratamiento en sala de emergencia a
} propósito de un caso

\author{
Julio Andres Alvarez Ortiz \\ julioalvarezuro@gmail.com \\ Servicio de Urología del Sanatorio del Salvador \\ (Córdoba - Argentina) \\ María Elena Quillupangui Caicedo \\ marielitaqb@yahoo.com \\ Posgradista de Medicina Familiar y Comunitaria \\ Universidad Central Del Ecuador \\ (Quito - Ecuador)
}

\section{Adriana Lisseth Escobar Morillo alemcitasun@yahoo.es \\ Especialista en Geriatría y Gerontología Pontificia Universidad Católica del Ecuador (Quito - Ecuador)}

\author{
Antonny David Aguas Salazar \\ anto2012as@hotmail.com \\ Urological Department of the Belgorod Regional Hospital \\ Belgorod National Research University \\ (Belgorod - Rusia) \\ Henry Balboa Chimborazo \\ henry77n@gmail.com \\ Ministerio de Salud Pública del Ecuador \\ Universidad de Cuenca \\ (Catamayo - Ecuador) \\ Andrés Ricardo Nacipucha Pazmiño \\ rikynp1997@hotmail.com \\ Hospital Francisco Icaza Bustamante \\ Universidad de Guayaquil \\ (Guayaquil - Ecuador) \\ Rosa Gabriela Valdiviezo Arias \\ 1896gabriela@gmail.com \\ Hospital Francisco Icaza Bustamante \\ Universidad de Guayaquil \\ (Guayaquil - Ecuador)
}

\section{RESUMEN}

Cólico renal es una de las patologías consultadas por emergencia, afectando a uno de cada once individuos, se estima que al menos el $50 \%$ de estos pacientes presentaran un nuevo episodio de dolor en los próximos 5 años y del 80 al $90 \%$ repetirá un episodio en 10 años, 
es importante conocer el manejo adecuado para casa caso sobre todo en fase de dolor agudo en la sala de emergencia por lo que se presenta un caso clínico resuelto adecuadamente. Objetivo: actualización bibliográfica sobre el manejo del cólico renal en sala de emergencia.

Materiales y métodos: se realizó búsqueda bibliográfica en las principales bases de datos disponibles en la actualidad, encontrando 3 revisiones sistemáticas, 2 guías prácticas de instituciones internacionales de urología, múltiples revisiones bibliográficas y reportes de caso, se seleccionó y cito las fuentes bibliográficas más relevantes y actualizadas.

Conclusiones y recomendaciones: Los analgésicos no esteroidales son los medicamentos de elección para manejo del dolor agudo del cólico renal, los analgésicos opioides son de segunda línea. Terapia expulsiva ha demostrado ser útil en cálculos mayores de $5 \mathrm{~mm}$ localizados en uréter distal. Anuria, infección urinaria y/o dolor refractario son criterios de internación y descompresión de urgencia mediante drenaje percutáneo o colocación de catéter ureteral.

Palabras clave: cólico renal; litiasis urinaria; cálculo renal. 


\title{
Renal colic: diagnosis and treatment in the emergency room on the subject of a case
}

\begin{abstract}
Renal colic is one of the most frequently consulted pathologies per emergency, affecting one in eleven individuals, it is estimated that at least $50 \%$ of these patients will present a new episode of pain in the next 5 years and 80 to $90 \%$ will repeat a episode in 10 years, it is important to know the proper management for each case, especially in the acute pain phase in the emergency room, for which a properly resolved clinical case is presented. Objective: bibliographic update on the management of renal colic in the emergency room. Materials and methods: a bibliographic search was carried out in the main databases currently available, finding 3 systematic reviews, 2 practical guides from international urology institutions, multiple bibliographic reviews and case reports, and the most relevant bibliographic sources were selected and cited. and updated.

Conclusions and recommendations: Non-steroidal analgesics are the drugs of choice for the management of acute pain in renal colic, opioid analgesics are second-line. Expulsive therapy has proven useful in stones larger than $5 \mathrm{~mm}$ located in the distal ureter. Anuria, urinary infection and / or refractory pain are criteria for hospitalization and emergency decompression by percutaneous drainage or placement of a ureteral catheter.
\end{abstract}

Key words: renal colic; urinary lithiasis; renal stone.

Artículo recibido: 10 Agosto. 2021 Aceptado para publicación: 07. Setiembre. 2021 Correspondencia: julioalvarezuro@gmail.com Conflictos de Interés: Ninguna que declarar 


\section{INTRODUCCIÓN}

Cólico renal es una de las patologías urológicas más frecuentes atendidas en las salas de emergencia a nivel mundial, en Estados Unidos se estima que 1 de cada 11 individuos presentará un episodio de cólico renal a causa de urolitiasis en toda su vida y al menos el $50 \%$ de estos pacientes repetirá un nuevo episodio de dolor en los próximos 10 años (Margaret S, 2014). En Europa la incidencia depende de factores geográficos, climáticos, étnicos y dietéticos, varía entre el 1\% al 20\%. (C. Türk, 2020)

Al ser una patología de presentación frecuente, es importante actualizar los conocimientos sobre el diagnóstico y manejo adecuado tanto del cólico renal como de la urolitiasis por lo que hemos decidido realizar una revisión bibliográfica a propósito de un caso clínico que se detalla a continuación.

\section{Caso clínico}

Se valora por sala de emergencia a paciente masculino de 33 años, sin antecedentes patológicos previos, refiere que varias horas previo a su valoración presenta de forma súbita y sin causa aparente dolor tipo cólico de gran intensidad localizado en fosa lumbar izquierda, que no mejora con cambios de posición, médicos de guardia administran analgésico endovenoso (diclofenaco E.V.) más antiespasmódico (hioscina E.V.), con lo cual mejora el dolor y es dado de alta con tamsulosina $0,4 \mathrm{mg}$ vía oral cada 24 horas. A los dos días reingresa por cólico renal persistente que se acompaña de síndrome febril, con sospecha de pielonefritis obstructiva se solicita pielotac donde se evidencia lito de 11 x $7 \mathrm{~mm}$ en tercio superior de uréter izquierdo (figura 1), laboratorio reporta leucocitosis con desviación a la izquierda, por lo que se decide su internación para tratamiento analgésico a base de Ketorolaco 30mg cada 8 horas endovenoso más dipirona $1 \mathrm{~g}$ cada 8 horas endovenoso, ceftriaxona $1 \mathrm{~g}$ cada 12 horas, se coloca catéter doble j, 7 días después es dado de alta y controlado por consultorio externo al décimo día de evolución con urocultivo negativo, se decide resolución quirúrgica mediante ureteroscopia más litotricia endoluminal y retiro de catéter, 24 horas posterior a intervención es dado de alta definitivamente. 

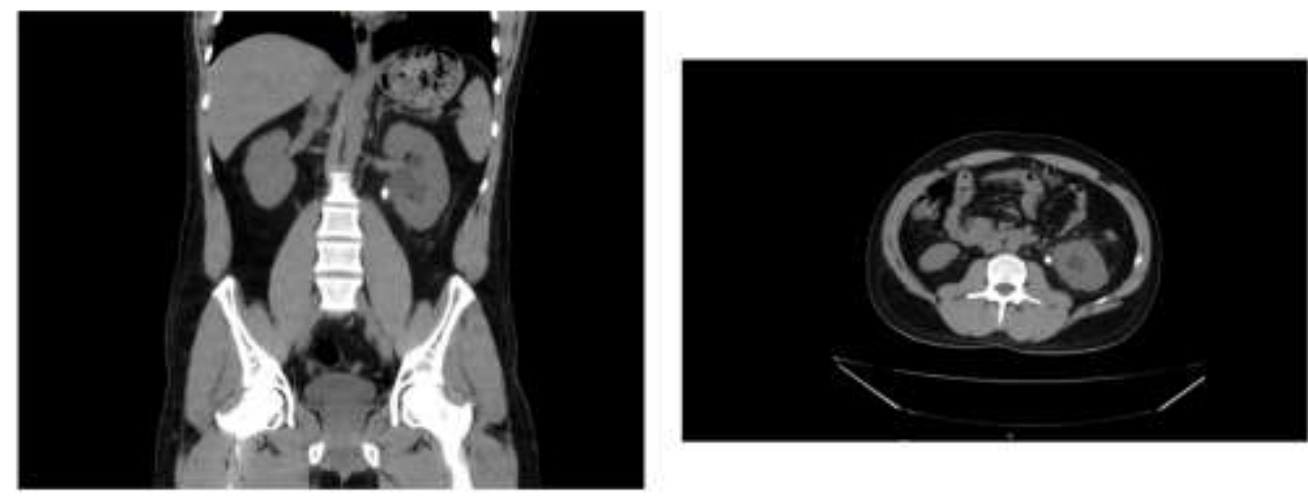

Figura 1. Se observa leve dilatación del sistema colector izquierdo, identificándose en unión pieloureteral imagen de litiasis. que mide $11 \mathrm{~mm}$ por $7 \mathrm{~mm}$, asociado a trabeculación de grasa perirrenal.

\section{MATERIALES Y MÉTODOS}

Se realizó búsqueda bibliográfica en las principales bases de datos disponibles en la actualidad, encontrando 3 revisiones sistemáticas, 2 guías prácticas de instituciones internacionales de urología, múltiples revisiones bibliográficas y reportes de caso, se seleccionó y cito las fuentes bibliográficas más relevantes y actualizadas.

\section{DISCUSIÓN}

Cólico renal es una de las patologías consultadas por emergencia, afectando a uno de cada once individuos, se estima que al menos el $50 \%$ de estos pacientes presentaran un nuevo episodio de dolor en los próximos 5 años y del 80 al $90 \%$ repetirá un episodio en 10 años (Margaret S, 2014) (Andreas Skolarikos, 2015).

Para comprender el manejo y evolución del cuadro clínico de dolor renal es importante conocer y comprender su fisiopatología del dolor y de la obstrucción urinaria. El dolor se produce por aumento de presión intraluminal debido a la obstrucción ureteral aguda se extiende desde les terminaciones nerviosas nociceptores localizadas en la submucosa y en la lámina propia de la cápsula renal peripiélica, la pelvis renal y en el uréter proximal. Estos impulsos nerviosos generados viajan hasta los segmentos medulares D11 - L2, la distribución por dermatomas del dolor renal es el resultado de una convergencia somatovisceral de la información neural recibida desde la médula espinal, por lo cual dolor se puede irradiar a cada órgano que comparte inervación con el tracto urinario (S. Esquena, 2006). 


\section{La obstrucción urinaria comprende de tres fases,}

La Primera Fase corresponde a las primeras 2 horas con aumento del flujo plasmático renal (FSR), acompaña de un aumento de la presión hidráulica tubular $(\mathrm{Pt})$ y de la compresión del sistema colector a causa de la obstrucción. En esta fase existe aumento de la Pt que es contrarrestado por un aumento de la tasa de filtrado glomerular (TFG) secundario a vasodilatación de arteriolas aferentes (preglomerulares), vasodilatación secundaria a liberación de PGE2 y Óxido Nítrico y aumento de la presión capilar glomerular.

La Segunda Fase corresponde a las siguientes 2-5 horas donde la Pt permanece elevada pero el FSR disminuye.

La Tercera Fase corresponde a las 5-24 horas de evolución en la cual se presenta mayor declinación del FSR junto con la Pt en paralelo y disminución de la compresión del sistema colector. Durante esta fase la Pt y el FSR declinan entre las 12 a $24 \mathrm{hs}$ de evolución, existe aumento de la resistencia de la arteriola aferente con disminución de la Pgc, redistribución del flujo de la periferia hacia zona yuxtaglomerular y vasoconstricción por acción de angiotensina II, endotelina y tromboxano A2. (Wein, 2015). Esta disminución de la presión tubular a expensas de la disminución del FSR durante la última fase explicaría la mejoría clínica de forma espontánea al cabo de algunas horas de inicio de los síntomas y explica la base del tratamiento analgésico que se tratara más adelante.

\section{Diagnóstico}

La presentación clínica se caracteriza por dolor súbito unilateral en fosa lumbar que se puede irradiar hacia flanco, región inguino genital y cara interna del muslo, puede también acompañarse de taquicardia, ansiedad y/o síntomas gastrointestinales como nausea y vomito, también puede acompañarse de síndrome febril el cual es un criterio de hospitalización y desobstrucción urgente al tratarse de una pielonefritis obstructiva.

La ecografía es el método de imagen más utilizado por su bajo costo y accesibilidad, sin emisiones de radiación ionizante, es de gran utilidad para identificar cálculos localizados en los calices, pelvis renal y de la unión vesicoureteral, presenta una sensibilidad del $45 \%$ y especificidad del 94\%, es el método de elección durante la gestación y edad pediátrica. (C. Türk, 2020)

La Tomografía Computarizada de Abdomen y pelvis sin contraste (Pielotac), tiene una sensibilidad del 95\%, es de gran utilidad para identificar cálculos de toda la via urinaria, 
facilita la localización, calcular el tamaño de los cálculos y decidir conducta terapéutica. (RICARDO SUSAETA, 2018) (C. Nicolaua, 2014)

\section{Tratamiento}

Una vez se establece un diagnóstico de cólico renal por presunta obstrucción urinaria, previo a la obtención de exámenes complementarios, lo primero que se debe realizar es un correcto tratamiento del dolor para lo cual se ha demostrado que los analgésicos no esteroideos (AINE) incluido el paracetamol son los analgésicos de elección durante la fase aguda (C. Türk, 2020), estos analgésico actúan inhibiendo producción de prostaglandinas con la consecuente disminución del filtrado glomerular logrando disminuir la presión hidráulica tubular (primera fase de la obstrucción urinaria) y consiguiendo también relajación del musculo liso ureteral, de esta manera se logra controlar el dolor el cual es el principal motivo de consulta. Se ha demostrado que los analgésicos opioides son igual de efectivos para el control del dolor renal, sin embargo, se asocian a efectos adversos como nausea y vomito, además con el uso de estos medicamentos usualmente se requiere dosis de rescate. (Sameer A, 2017)

El uso de antiespasmódicos no ha presentado evidencia en el control del dolor por si solo o asociado a otros tipos de analgésicos, por lo que se contraindica el uso de estos medicamentos en el tratamiento del cólico renal. (Afshar K, Jafari S, 2015)

Terapia expulsiva con el uso de alfa bloqueantes es controversial, pero se aconseja el uso de tamsulosina en cálculos del uréter distal mayor a 5mm. ((Margaret S, 2014)) (Astroza Eulufi,2019)

En todos los pacientes con dolor refractario que no responde a analgesia, aquellos que presente anuria o signos de infección, se debe ofrecer terapia para desobstrucción de la vía urinaria de urgencia mediante drenaje percutáneo o colocación de catéter ureteral, se debe recolectar muestra para urocultivo e iniciar tratamiento antibiótico inmediatamente. (C. Türk, 2020)

\section{CONCLUSIONES Y RECOMENDACIONES.}

- El caso clínico que se presento fue resuelto adecuadamente según bibliografía disponible.

- Los analgésicos no esteroidales son los medicamentos de elección para manejo del dolor agudo del cólico renal, los analgésicos opioides son de segunda línea. 
- Terapia expulsiva ha demostrado ser útil en cálculos mayores de $5 \mathrm{~mm}$ localizados en uréter distal.

- Anuria, infección urinaria y/o dolor refractario son criterios de internación y descompresión de urgencia mediante drenaje percutáneo o colocación de catéter ureteral.

- En todos los casos de cólico renal deben ser derivados a control con urología.

\section{REFERENCIAS BIBLIOGRAFICAS}

Margaret S. Pearle, David S. Goldfarb, Et. Col. (2014), Medical Management Of Kidney Stones: Aua Guideline, Juro, Vol. 192, 316-324 Http://Dx.Doi.Org/10.1016/J.Juro.2014.05.006

C. Türk (Chair), A. Neisius, A. Petrik, C. Seitz, A. Skolarikos (Vice-Chair), K. Thomas, (2020), Eau Guidelines On Urolithiasis, European Association Of Urology. Disponible En: Https://Uroweb.Org/Wp-Content/Uploads/Eau-Guidelines-On$\underline{\text { Urolithiasis-2020v5.Pdf }}$

S. Esquena, F. Millán Rodríguez, F.M. Sánchez-Martín, F. Rousaud Barón, F. Marchant,

H. Villavicencio Mavrich, (2006), Cólico Renal: Revisión De La Literatura Y Evidencia Científica, Actas Urol Esp; 30 (3): 268-280

Wein, (2015), Campbell - Walsh Urologia, 10 Edición, Tomo Ii, Editorial Panamericana Andreas Skolarikos, Michael Straub, Thomas Knoll, Kemal Sarica, Christian Seitz, Ales Petř́ik, Christian Türk, (2015), Metabolic Evaluation And Recurrence Prevention For Urinary Stone Patients: Eau Guidelines. Disponible En: Https://Doi.Org/10.1016/J.Eururo.2014.10.029

Sameer A. Pathan, Biswadev Mitra, Peter A. Cameron, (2017), A Systematic Review And Meta-Analysis Comparing The Efficacy Of Nonsteroidal Anti-Inflammatory Drugs, Opioids, And Paracetamol In The Treatment Of Acute Renal Colic, $\begin{array}{llllll}\text { European } & \text { Urology } & 73 & \text { (2018) } & 583 \quad & -\end{array}$ Https://Doi.Org/10.1016/J.Eururo.2017.11.001

Afshar K, Jafari S, Marks Aj, Eekhari A, Macneily Ae, (2015), Nonsteroidal AntiInflammatory Drugs (Nsaids) And Non-Opioids For Acute Renal Colic (Review), Cochrane Database Of Systematic Reviews, Issue 6. Art. No.: Cd006027. Https://Doi.Org/10.1002/14651858.Cd006027.Pub2 
Astroza Eulufi, Gastón; Sarrás Jadue, Miguel; Bettancourt Guglielmetti, Cristóbal; Lara Hernández, Bárbara; Neira Soto, Rodrigo; Aguilera Fuenzalida, Pablo, (2019), Efectividad Del Tratamiento Médico Expulsivo Con El Bloqueador Alfa Tamsulosina En Pacientes Adultos Que Consultan Por Cálculo Ureteral Distal En Un Servicio De Urgencias Chileno / Effectiveness Of Medical Expulsive Therapy With The Alfa-Adrenergic Blocker Tamsulosin For Distal Ureterolithiasis In Adults Attended In An Emergency Department In Chile, Emergencias: Revista De La Sociedad Española De Medicina De Urgencias Y Emergencias, Issn 11376821, Vol. 31, Nº 6 (Diciembre), 2019, Págs. 404-406.

Dr. Ricardo Susaeta, Dr. David Benavente, Dr. Fernando Marchant, Dr. Renato Gana, (2018), Diagnóstico Y Manejo De Litiasis Renales En Adultos Y Niños, [Rev. Med. Clin. Condes; 29(2) 197-212]

Christian Türk, Ales Petrık , Kemal Sarica, Christian Seitz, Andreas Skolarikos, Michael

Straub, Thomas Knoll, Eau Guidelines On Diagnosis And Conservative Management Of Urolithiasis, European Urology 69 (2016) 468 -474, Http://Dx.Doi.Org/10.1016/J.Eururo.2015.07.040

Francisco Javier Ancizu, Fernando Díez-Caballero, (2018), Colico Renal, Guías De Actuación En Urgencias, Clinica Universidad De Navarra. Disponible En: File:///C:/Users/Asus/Downloads/Guia-Actuacion-Colico-Renal\%20(1).Pdf

M.A. Aibar-Arregui , M. Matía-Sanz, R. Pelay-Cacho, M.J. Igúzquiza-Pellejero, M.P. Martín-Fortea , L. Clavel-Conget, C. Gómez-Del Valle, F.J. Ruiz-Ruiz, B. SierraBergua, (2010), Manejo Del Cólico Renal En Urgencias De Un Hospital De Tercer Nivel, An. Sist. Sanit. Navar.; 33 (2): 145-154

C. Nicolaua, R. Salvador, J.M. Artigas, (2014), Manejo Diagnóstico Del Cólico Rena, Rx-771; Http://Dx.Doi.Org/10.1016/J.Rx.2014.11.003

J. Lorca Álvaro, I. Laso García, M. Santiago González, F.J. Burgos Revilla, (2019),

Síndrome De Dolor Lumbar Agudo No Traumático De Origen Renal: Cólico Renoureteral, Medicine. 2019;12(87):5109-16.

Hermida Pérez Ja, Bermejo Hernández A, Hernández Guerra Js, Sorbenes Gutiérrez Rj, (2013), Tratamiento Del Cólico Nefrítico En Urgencias: Nuestra Experiencia, Med Gen Y Fam (Digital);2(3):67-75. 
R. Martínez Pérez, (2012), Revisión Del Tratamiento Farmacológico De La Urolitiasis, Fap.;10(1):18-2.

Dean Assimos, Md; Amy Krambeck, Md; Nicole L. Miller, Md, Et Al, (2016), Surgical Management Of Stones: American Urological Association/ Endourological Society Guideline, American Urological Association (Aua) Endourological Society Guideline.

Sílvia Ferrer Moreta, Eva Bellerino Serrano, David Pérez Morales, (2015), Litiasis Renal: Criterios De Estudio, Derivación Y Tratamiento, Fmc.;22(6):301-11

Nie Zhen, Chen De-Sheng, Yan Yan-Jun, Lin Hua, (2021), The American Journal Of Emergency Medicine Volume 43, Pages 12-16

Christopher L. Moore, (2019), Imaging In Suspected Renal Colic: Systematic Review Of The Literature And Multispecialty Consensus, Annals Of Emergency Medicine, Volume 74, Issue 3, Pages 391-399 